\title{
Acute Isolated Bilateral Mydriasis: Case Reports and Review of the Literature
}

\author{
Hiromasa Sato Kosuke Naito Takao Hashimoto \\ Department of Neurology, Aizawa Hospital, Matsumoto, Japan
}

Key Words

Fisher syndrome · Bickerstaff brainstem encephalitis · Anti-GQ1b antibody · Internal ophthalmoplegia - Mydriasis - Anti-GQ1b IgG antibody syndrome

\begin{abstract}
Isolated bilateral internal ophthalmoplegia is a rare and problematic condition affecting activities of daily living. Herein, we describe the cases of 2 patients with postinfectious isolated bilateral internal ophthalmoplegia, i.e., mydriasis without external ophthalmoplegia. One patient demonstrated no other neurological symptom, while the other patient showed mild gait ataxia. Magnetic resonance imaging revealed no abnormal findings in the brain or brainstem. Light-near dissociation of the pupils was not recognized in either patient, and supersensitivity to dilute pilocarpine was observed in 1 of the 2 patients. An increased titer of the anti-GQ1b IgG antibody was noted in 1 patient. A review of the literature revealed five similar cases; the symptomatic characteristics, ratio of positive anti-GQ1b IgG antibody, and effective treatment are discussed.

(c) 2014 S. Karger AG, Basel
\end{abstract}

\section{Introduction}

Acute bilateral isolated internal ophthalmoplegia, i.e., mydriasis without external ophthalmoplegia, is a rare manifestation. Postinfectious internal ophthalmoplegia can be associated with botulism, syphilis, Fisher syndrome (FS), Bickerstaff brainstem encephalitis (BBE), and Guillain-Barré syndrome (GBS); it is usually associated with external ophthalmoplegia and other neurological symptoms. Only a few cases of isolated internal ophthalmoplegia have been reported, and all the tested cases have been shown to be associated with the anti-GQ1b IgG antibody [1-5]. Herein, we describe the cases of 2 patients with postinfectious isolated bilateral internal ophthalmoplegia and review the previously reported cases.

Takao Hashimoto, MD, PhD

Center for Neurological Diseases, Aizawa Hospital

2-5-1 Honjo, Matsumoto 390-8510 (Japan)

E-Mail sinke-dr@ai-hosp.or.jp 
Sato et al.: Acute Isolated Bilateral Mydriasis: Case Reports and Review of the Literature

\section{Case Reports}

\section{Patient 1}

A 28-year-old man experienced upper respiratory symptoms and presented with photophobia 5 days later. Examination on day 4 of photophobia onset revealed that he was afebrile and fully alert. He had no eye pain. His distance visual acuity, intraocular pressure, and funduscopic findings were normal. His near visual acuity was not tested. His pupils were isocoric but remarkably mydriatic and $6.5 \mathrm{~mm}$ in diameter under the normal light of the room. There was no limitation to ocular movement, including accommodation. Light and accommodation reflexes were absent. There was no evidence of facial or oropharyngeal palsy. There was no limb weakness or ataxia, but the patient's deep tendon reflexes were decreased in the upper extremities and absent in the lower extremities. His plantar responses were flexor. His sensations of touch, pain, and vibration were normal. Instillation of $0.1 \%$ pilocarpine on the right side caused minimal pupillary constriction, whereas instillation of $2 \%$ pilocarpine caused pupillary constriction to $3.0 \mathrm{~mm}$. His pupillary response to epinephrine was not tested. Routine hematological examinations were unremarkable. The serum IgG anti-GQ1b antibody titer was increased to 7.5 (normal: $<1.0$ ), while the serum IgG anti-GM1 antibody titers were not increased. Screening for autoantibodies, antinuclear antibodies, anti SS-A, and anti SS-B was negative. The cerebrospinal fluid showed $23 \mathrm{mg} / \mathrm{dl}$ of protein (normal: $10-40 \mathrm{mg} / \mathrm{dl}$ ), and the white cell count was normal. Brain magnetic resonance imaging (MRI) showed no abnormalities. The patient was treated with a 3-day course of intravenous methylprednisolone, which was followed by oral prednisolone $(40 \mathrm{mg} /$ day). His pupil size gradually diminished and returned to normal on day 18.

\section{Patient 2}

A 29-year-old man experienced diarrhea and presented with photophobia a week later. Neurological examination on day 5 of photophobia onset revealed dilated pupils, which were $7.5 \mathrm{~mm}$ in diameter, on both sides but without limitations of ocular movement, including accommodation. His distance visual acuity was normal, and his near visual acuity was not tested. Both light and accommodation reflexes were absent. The funduscopic findings were normal. He showed no limb ataxia. The tandem gait test revealed slight ataxia, but the patient's gait was almost normal. His deep tendon reflexes were symmetric and normal in the upper extremities and slightly exaggerated in the lower extremities. The plantar responses were flexor. His sensations of touch, pain, and vibration were normal. Instillation of $2 \%$ of pilocarpine caused pupillary constriction to approximately $3.0 \mathrm{~mm}$. Diluted pilocarpine was not used. The pupillary response to epinephrine was not tested. Routine hematological examinations were unremarkable. The serum IgG anti-GQ1b antibody titer was not determined. Brain MRI showed no abnormalities. The patient was treated with a 3day course of intravenous methylprednisolone $(1,000 \mathrm{mg} /$ day), which was followed by oral prednisolone $(40 \mathrm{mg} /$ day). The gait ataxia disappeared within a week. The photophobia gradually improved, and the pupil size returned to normal, with normal light reflex noted on day 98.

\section{Discussion}

The main clinical feature of our patients was postinfectious bilateral internal ophthalmoplegia without external ophthalmoplegia. Patient 2 demonstrated no other neurological 
symptom, while patient 1 showed mild gait ataxia. MRI revealed no abnormality in the brain or brainstem. Botulism was excluded because there was no history of causative food intake and no cranial nerve involvement, other than in the pupils. Based on these findings, a postinfectious autoimmune etiology was suggested. In patient 1 , an assay for antiganglioside antibodies revealed an increased titer of IgG anti-GQ1b $(7.5$, normal: $<0.4)$.

In the literature, we found five cases of patients with presumed autoimmune isolated bilateral internal ophthalmoplegia (table 1) [1-5]. We analyzed the clinical features of all 7 patients, including our 2 patients. Their ages ranged from 21 to 53 years (mean, 33.9 years), and the male-female ratio was 5:2. The male preponderance was in contrast to Adie's syndrome in which the majority of patients are female [6]. Light-near dissociation of the pupils was recognized in only 1 of 6 patients examined. Supersensitivity to dilute pilocarpine was noted in 2 of the 3 patients assessed. Light-near dissociation results from either afferent pupillary pathway dysfunction or a posterior ciliary ganglion lesion, while supersensitivity to dilute pilocarpine suggests damage to the ciliary ganglion or to the postganglionic parasympathetic fibers. These findings suggest that the responsible lesion sites may be located in the ciliary ganglion and surrounding areas and that the degree of postganglionic fiber damage may have varied. Deep tendon reflexes were decreased or absent in 4 patients, exaggerated in 2 , and normal in 1 . The 2 patients with exaggerated tendon reflexes did not demonstrate extensor plantar responses. Mild ataxia was present in 3 of 7 patients.

An increased titer of the IgG anti-GQ1b antibody was revealed in 6 patients. High sensitivity of anti-GQ1b antibodies has been confirmed in FS [7, 8], while an increased titer of IgG anti-GQ1b has been reported in BBE, GBS, and acute ophthalmoparesis [9]. It has been proposed that these diseases fall under the category of anti-GQ1b IgG antibody syndromes [9]. Among the symptoms of these diseases, ophthalmoplegia and ataxia have been shown to be closely correlated with the IgG anti-GQ1b antibody $[9,10]$. Internal ophthalmoplegia with increased anti-GQ1b IgG antibody titers has been reportedly observed in 7\% of patients with acute ophthalmoparesis, $37 \%$ of patients with FS, and $42 \%$ of patients with BBE [9]. Internal ophthalmoplegia has also been observed in a few patients with GBS, especially in the severe cases [11]. These findings suggest that isolated internal ophthalmoplegia may be a variant of anti-GQ1b IgG antibody syndrome. Abundant anti-GQ1b antibodies exist in the paranodal regions of the extraocular cranial nerves [10], and internal ophthalmoplegia has been speculated to be caused by ciliary ganglionic involvement [1]. It remains elusive whether isolated internal ophthalmoplegia occurs in association with negative IgG anti-GQ1b antibody. The different deep tendon reflex responses observed in the 7 patients suggest that internal ophthalmoplegia associated with hyporeflexia or areflexia is more like FS, while internal ophthalmoplegia associated with hyperreflexia is more like BBE.

The natural course of isolated ophthalmoplegia is benign and self-limited. Four patients who underwent steroid pulse treatment showed recovery after 13 days to 3 months. On the other hand, the 3 patients who underwent no treatment showed recovery in 20 and 22 days and 4 months. This small difference in the recovery periods suggests that steroid therapy is not beneficial, which is also the case in FS, BBE, and GBS. Because internal ophthalmoplegia could lead to severe photophobia and blurred vision for some patients, the efficacy of other immunological strategies, such as plasmapheresis or intravenous immunoglobulin, should be investigated.

\section{Disclosure Statement}

The authors have no conflicts of interest to disclose. 
Sato et al.: Acute Isolated Bilateral Mydriasis: Case Reports and Review of the Literature

\section{References}

1 Radziwill AJ, Steck AJ, Borruat FX, Bogousslavsky J: Isolated internal ophthalmoplegia associated with IgG anti-GQ1b antibody. Neurology 1998;50:307.

-2 Yuki N, Koga M, Hirata K: Isolated internal ophthalmoplegia associated with Immunoglobulin G anti-GQ1b antibody. Neurology 1998;51:1515-1516.

-3 Mori M, Takeshima T, Yuki N, Nakashima K: A case of anti-GQ1b-positive atypical Fisher syndrome with internal ophthalmoplegia but without external ophthalmoplegia. Rinsho Shinkeigaku 2000;40:887-890.

4 Sugita A, Yanagisawa T, Kamo T, Takahashi Y, Yuki N: Internal ophthalmoplegia with anti-GQ1b IgG antibody. J Neurol 2002;249:1475-1476.

5 Fleury V, Aqallal A, Lagrange E, Besson G, Caudie C: Acute bilateral mydriasis associated with anti-GQ1b antibody. J Clin Neurosci 2010;17:514-515.

-6 Bremner FD, Smith SE: Bilateral tonic pupils: Holmes Adie syndrome or generalised neuropathy? Br J Ophthalmol 2007;91:1620-1623.

7 Yuki N, Sato S, Tsuji S, Ohsawa T, Miyatake T: Frequent presence of anti-GQ1b antibody in Fisher's syndrome. Neurology 1993;43:414-417.

8 Mori M, Kuwabara S, Yuki N: Fisher syndrome: clinical features, immunopathogenesis and management. Expert Rev Neurother 2012;12:39-51.

9 Odaka M, Yuki N, Hirata K: Anti-GQ1b IgG antibody syndrome: clinical and immunological range. J Neurol Neurosurg Psychiatry 2001;70:50-55.

10 Chiba A, Kusunoki S, Obata H, Machinami R, Kanazawa I: Serum anti-GQ1b IgG antibody is associated with ophthalmoplegia in Miller Fisher syndrome and Guillain-Barré syndrome: clinical and immunohistochemical studies. Neurology 1993;43:1911-1917.

11 Hung JC, Appleton RE: Iridoplegia in severe Guillain-Barré syndrome. Arch Dis Child 1997;77:91-95.

Table 1. Case reports of patients with isolated bilateral mydriasis manifesting with no external ophthalmoplegia

\begin{tabular}{|c|c|c|c|c|c|c|c|c|}
\hline Authors & $\begin{array}{l}\text { Age/ } \\
\text { sex }\end{array}$ & $\begin{array}{l}\text { Light-near } \\
\text { dissociation }\end{array}$ & $\begin{array}{l}\text { Pupillary } \\
\text { super-sensitivity }\end{array}$ & $\begin{array}{l}\text { Deep tendon } \\
\text { reflex }\end{array}$ & Ataxia & $\begin{array}{l}\text { IgG } \\
\text { anti-GQ1b } \\
\text { antibody }\end{array}$ & Treatment & $\begin{array}{l}\text { Recovery } \\
\text { time }\end{array}$ \\
\hline Radziwill et al. [1] & $33 / \mathrm{M}$ & + & + & normal & + & + & none & 20 days \\
\hline Yuki et al. [2] & $36 / \mathrm{M}$ & - & not described & decreased & - & + & none & 22 days \\
\hline Mori et al. [3] & $21 / \mathrm{M}$ & - & not described & decreased & + & + & $\begin{array}{l}\text { IAPP steroid } \\
\text { pulse }\end{array}$ & 13 days \\
\hline Sugita et al. [4] & $53 / F$ & - & + & exaggerated & + & + & steroid pulse & $>2$ months \\
\hline Fleury et al. [5] & $37 / F$ & not described & not described & decreased & - & + & none & $<4$ months \\
\hline \multirow[t]{2}{*}{ Our cases } & $28 / \mathrm{M}$ & - & - & decreased & - & + & steroid pulse & 18 days \\
\hline & $29 / \mathrm{M}$ & - & not tested & exaggerated & + & not tested & steroid pulse & 3 months \\
\hline
\end{tabular}

IAPP = Immunoadsorption plasmapheresis. 